\title{
A NOTE ON NOETHERIAN ORDERS IN ARTINIAN RINGS
}

\author{
by A. W. CHATTERS
}

(Received 9 November, 1977)

Throughout this note, rings are associative with identity element but are not necessarily commutative. Let $R$ be a left and right Noetherian ring which has an Artinian (classical) quotient ring. It was shown by S. M. Ginn and P. B. Moss [2, Theorem 10] that there is a central idempotent element $e$ of $R$ such that $e R$ is the largest Artinian ideal of $\boldsymbol{R}$. We shall extend this result, using a different method of proof, to show that the idempotent $e$ is also related to the socle of $R / N$ (where $N$, throughout, denotes the largest nilpotent ideal of $R$ ) and to the intersection of all the principal right (or left) ideals of $R$ generated by regular elements (i.e. by elements which are not zero-divisors). There are many examples of left and right Noetherian rings with Artinian quotient rings, e.g. commutative Noetherian rings in which all the associated primes of zero are minimal together with full or triangular matrix rings over such rings. It was shown by L. W. Small that if $R$ is any left and right Noetherian ring then $R$ has an Artinian quotient ring if and only if the regular elements of $R$ are precisely the elements $c$ of $R$ such that $c+N$ is a regular element of $R / N$ (for further details and examples see [5] and [6]). By the largest Artinian ideal of $R$ we mean the sum of all the Artinian right ideals of $R$, and it was shown by T. H. Lenagan in [3] that this coincides in any left and right Noetherian ring $R$ with the sum of all the Artinian left ideals of $R$.

Before proving the main theorem we shall give for the reader's convenience the proof of a basic result which is a consequence of Goldie's theorem but which does not seem to be readily available in the literature. Let $R$ be a semi-prime right Noetherian ring and let $S$ be the right socle of $R$; we shall show that $S$ is generated by a central idempotent element of $R$ (in fact this result is true for any semi-prime right Goldie ring). There is a right ideal $K$ of $R$ such that $S \cap K=0$ and $S+K$ is an essential right ideal of $R$. We have $K S=0$ because $S$ is a two-sided ideal of $R$. By Goldie's theorem there is a regular element $c$ of $R$ such that $c \in S+K$. Thus $c=s+k$ for some $s \in S$ and $k \in K$. Because $S$ and $c S$ have the same length, as right $R$-modules, we have $S=c S$. Hence $s=c t$ for some $t \in S$. We have $c t=(s+k) t=c t^{2}+k t=c t^{2}$ so that $t=t^{2}$. Also if $u \in S$ then $c u=(c t+k) u=$ $c t u$ so that $u=t u$. Therefore $S=t R$. Finally, we have $(S(1-t))^{2}=S(1-t) . t S(1-t)=0$ so that $S(1-t)=0$ from which it follows easily that $S=R t$ and that $t$ is central.

THEOREM 1. Let $R$ be a left and right Noetherian ring which has an Artinian quotient ring. Then there is a central idempotent element $e$ of $R$ such that

(i) (Ginn and Moss) $e R$ is the largest Artinian ideal of $R$,

(ii) $(e R+N) / N$ is the socle of $R / N$, and

(iii) $e R=\bigcap c R=\bigcap R c$, where $c$ ranges over the regular elements of $R$.

Proof. Let ${ }^{*}$ denote image in $R^{*}=R / N$ and let $S$ denote the socle of $R^{*}$. Because idempotents can be lifted over $N$, and because $S$ is the socle of the semi-prime Noetherian ring $R^{*}$, we have $S=e^{*} R^{*}=R^{*} e^{*}$ for some idempotent element $e$ of $R$. Let $A$ be the

Glasgow Math. J. 20 (1979) 125-128. 
largest Artinian ideal of $R$, and let $G=\bigcap c R$ and $H=\bigcap R c$, where $c$ ranges over the regular elements of $R$. Because $A$ is Artinian both as a right $R$-module and as a left $R$-module, we have $A=c A=A c$ for every regular element $c$ of $R$, so that $A \subseteq G \cap H$. We shall show in the next paragraph that $H$ is a two-sided ideal of $R$ and that $H$ is a divisible right $R$-module. It will then follow from Lemma (A) that $H \subseteq \operatorname{Re} \cap A$. It will follow from Lemma (B) that $e \in H$. The proof of Theorem 1 is then completed by combining these facts and using left-right symmetry.

Let $h \in H, r \in R$, and let $c$ be a regular element of $R$. Because $R$ has a left quotient ring and hence satisfies the left Ore condition we have $x c=d r$ for some $x \in R$ and regular element $d$ of $R$. But $h \in H$, so that $h=y d$ for some $y \in R$. Thus $h r=y d r=y x c \in R c$. Therefore $h r \in H$, from which it follows that $H$ is a two-sided ideal of $R$. We also have $h=z c$ for some $z \in R$. Let $c^{\prime}$ be a regular element of $R$; then $h \in R c^{\prime} c$ so that $h=z^{\prime} c^{\prime} c$ for some $z^{\prime} \in R$. Thus $z^{\prime} c^{\prime} c=z c$, so that $z=z^{\prime} c^{\prime}$. Hence $z \in R c^{\prime}$ for every regular element $c^{\prime}$ of $R$, i.e. $z \in H$. Thus $h \in H c$, which gives $H=H c$. Therefore $H$ is a divisible right $R$-module.

LeMMA (A). With the above notation, let $M$ be a finitely-generated torsion-free divisible right $R$-module. Then $M$ is Artinian and $M=M e$.

Proof. Let $Q$ be the quotient ring of $R$. We can give $M$ the structure of a right $Q$-module in a natural way. Set $X=M N^{i} / M N^{i+1}$, where $i$ is a non-negative integer. It suffices to show that $X$ is Artinian and $X=X e$. We may suppose that $X \neq 0$. By Theorem 1.9 of [5] we have $N Q=Q N$. Hence $M N^{i}, M N^{i+1}$, and $X$ are all right $Q$-modules. Because $X N=0$ we can give $X$ the structure of a right $R^{*}$-module, and, as such, $X$ is finitely-generated, torsion-free and divisible. Let $T$ be the quotient ring of $R^{*}$. We can make $X$ into a right module over the semi-simple Artinian ring $T$. Let $U$ be the sum of all the minimal right ideals of $T$ which are isomorphic to submodules of $X$. Then there is an ideal $V$ of $T$ such that $T=U \oplus V$ and $X V=0$. But $X$ and $U$ have the same isomorphism types of simple $T$-submodules. Therefore $U$ is contained in the direct sum of a finite number of copies of $X$. Hence $U$ is a finitely-generated right $R^{*}$-module and it follows that $U \subseteq R^{*}$. Thus an $R^{*}$-submodule of $X$ is also a $U$-submodule and hence a $T$ submodule, so that $X$ is Artinian as an $R^{*}$-module. It follows that $U$ is Artinian as an $R^{*}$-module, i.e. $U$ is contained in the socle $R^{*} e^{*}$ of $R^{*}$. Therefore $U e^{*}=U$, giving $X e^{*}=X$ and hence $X=X e$.

Lemma (B). With the above notation, $e \in H$.

Proof. It is enough to show that $e \in R c$, where $c$ is an arbitrary regular element of $R$. Hence it is enough to show that $e M=0$ for any torsion left $R$-module $M$ (in particular for $M=R / R c$ ). Also we may suppose that $N M=0$ and make $M$ into a left $R^{*}$-module. Let $m \in M$; then $d m=0$ for some regular element $d$ of $R$. But $d^{*}$ is a regular element of $R^{*}$, so that $R^{*} e^{*} d^{*}=R^{*} e^{*}$. Therefore $R^{*} e^{*} m=R^{*} e^{*} d^{*} m=R^{*} e^{*} d m=0$, so that $0=e^{*} m=$ $e m$, which completes the proof.

We shall now give two applications of Theorem 1. The first is to derive J. C. Robson's 
decomposition theorem for Noetherian rings, and the second is to give a shorter proof of the decomposition theorem for Noetherian p.p. rings.

THEOREM 2. (Robson [4, Theorem 1]). Let $R$ be a left and right Noetherian ring such that $N=c N=N c$ for every element $c$ of $R$ such that $c+N$ is regular in $R / N$. Then $R$ is the direct sum of an Artinian ring and a semi-prime ring.

Proof. We may suppose that $R$ is indecomposable and not Artinian. Let $c$ be an element of $R$ such that $c+N$ is regular in $R / N$. Multiplication by $c$ induces a surjective endomorphism of the Noetherian module $N$ and hence is an automorphism of $N$. It now follows easily that $c$ is regular. Therefore $R$ has an Artinian quotient ring by Small's theorem. In the notation of Theorem 1 , we have $e=0$ and $N \subseteq e R$ by Theorem 1 (iii). Therefore $N=0$.

Theorem 3. ([1, Theorem 3.1]). Let $R$ be a left and right Noetherian p.p. ring. Then $R$ is a direct sum of Artinian rings and prime rings.

Proof. It was shown by L. W. Small that $R$ has an Artinian quotient ring [7, Theorem 2]. We may suppose that $R$ is indecomposable and not Artinian.

Because $N$ has non-zero right annihilator and the left singular ideal of $R$ is zero, $N$ is not an essential left ideal of $R$. Therefore there is a prime ideal $P$ of $R$ which is not an essential left ideal. We shall prove that $P=0$. There is a non-zero left ideal $L$ of $R$ such that $P \cap L=0$, and it follows that $P$ is the left annihilator of $L$. Therefore $P=R(1-f)$ for some idempotent element $f$ of $R$ [8, Theorem 1]. Let $g$ be a primitive idempotent of the ring $f R f$, let $x \in P$, and let $c$ be a regular element of $R$. Set

$$
y=f-g+g x-(1-f) c .
$$

Note that $R(1-f)$ and $f R$ are two-sided ideals of $R$. We have $y g=0$. If $y$ has zero left annihilator then $R y$ is an essential left ideal and since $R$ is left non-singular it follows that $g=0$. Therefore there is a non-zero idempotent element $h$ of $R$ such that $h y=0$ (because $R$ is a p.p. ring). We have hyf $=0$, so that $h f=h g$. Set $j=h f$; then $j g=j=j^{2}$ and $j \in f R f$. But $g$ is primitive, and $g j$ and $g-g j$ are orthogonal idempotents in $f R f$. Therefore $g j=0$ or $g j=g$. If $g j=0$ then $0=j g j=j=h f$, so that $0=h y=-h c$, which is not so. Therefore $g j=g$, i.e. $g h f=g$. Hence $0=g h y=g x-g h(1-f) c$. Thus $g x \in R c$. Hence $g P \subseteq e R$, where $e$ is as in Theorem 1. But $R$ is indecomposable and not Artinian, so that $e=0$. Therefore $g P=0$. But $f$ is a sum of idempotents such as $g$. Therefore $f P=0$, i.e. $f R(1-f)=0$. Thus $f R(1-f)=0=(1-f) R f$, so that $f$ is a central idempotent element of $R$. Therefore $f=0$ or $f=1$. But $f \neq 0$ because $R(1-f)$ is a prime ideal of $R$. Therefore $f=1$, giving $P=0$.

There are standard examples in the literature which can be used to show that the conclusion of Theorem 1 does not hold for an arbitrary left and right Noetherian ring or for an arbitrary right Noetherian ring which has a right Artinian right quotient ring. An example of the first type is the ring $T$ of [9] which is left and right Noetherian, has no non-trivial central idempotents, and in which the largest Artinian ideal is a proper ideal. In [10] there are examples of right Noetherian rings which have right Artinian right 
quotient rings, which are not themselves right Artinian, which have non-zero Artinian right ideals, and which have no non-trivial central idempotents.

\section{REFERENCES}

1. A. W. Chatters, Two results on p.p. rings, Comm. Algebra 4 (1976), 881-891.

2. S. M. Ginn and P. B. Moss, A decomposition theorem for Noetherian orders in Artinian rings, Bull. London Math. Soc. 9 (1977), 177-181. 499-500.

3. T. H. Lenagan, Artinian ideals in Noetherian rings, Proc. Amer. Math. Soc. 51 (1975),

4. J. C. Robson, Decomposition of Noetherian rings, Comm. Algebra 1 (1974), 345-349.

5. L. W. Small, Orders in Artinian rings, J. Algebra 4 (1966), 13-41.

6. L. W. Small, Orders in Artinian rings II, J. Algebra 9 (1968), 266-273.

7. L. W. Small, Hereditary rings, Proc. Nat. Acad. Sci. U.S.A. 55 (1966), 25-27.

8. L. W. Small, Semi-hereditary rings, Bull. Amer. Math. Soc. 73 (1967), 656-658.

9. L. W. Small, On some questions in Noetherian rings, Bull. Amer. Math. Soc. 72 (1966), 853-857.

10. L. W. Small, An example in Noetherian rings, Proc. Nat. Acad. Sci. U.S.A. 54 (1965), 1035-1036.

SchoOl of Mathematics

UNIVERSTTY WALK.

BRISTOL BS8 1TW 